\title{
Small-molecule correctors of defective $\Delta$ F508-CFTR cellular processing identified by high-throughput screening
}

\author{
Nicoletta Pedemonte, ${ }^{1,2}$ Gergely L. Lukacs, ${ }^{3}$ Kai Du, ${ }^{3}$ Emanuela Caci, ${ }^{2}$ \\ Olga Zegarra-Moran, ${ }^{2}$ Luis J.V. Galietta, ${ }^{2}$ and A.S. Verkman ${ }^{1}$
}

'Departments of Medicine and Physiology, UCSF, San Francisco, California, USA. 'Laboratorio di Genetica Molecolare, Istituto Giannina Gaslini, Genova, Italy. ${ }_{3}^{3}$ Program in Cell and Lung Biology, Hospital for Sick Children Research Institute, and University of Toronto, Toronto, Ontario, Canada.

\begin{abstract}
The most common cause of cystic fibrosis (CF) is deletion of phenylalanine $508(\Delta \mathrm{F} 508)$ in the CF transmembrane conductance regulator (CFTR) chloride channel. The $\Delta$ F508 mutation produces defects in folding, stability, and channel gating. To identify small-molecule correctors of defective cellular processing, we assayed iodide flux in $\triangle F 508$-CFTR-transfected epithelial cells using a fluorescent halide indicator. Screening of 150,000 chemically diverse compounds and more than 1,500 analogs of active compounds yielded several classes of $\triangle$ F508-CFTR correctors (aminoarylthiazoles, quinazolinylaminopyrimidinones, and bisaminomethylbithiazoles) with micromolar potency that produced greater apical membrane chloride current than did low-temperature rescue. Correction was seen within 3-6 hours and persisted for more than 12 hours after washout. Functional correction was correlated with plasma membrane expression of complex-glycosylated $\triangle$ F508-CFTR protein. Biochemical studies suggested a mechanism of action involving improved $\triangle F 508$-CFTR folding at the ER and stability at the cell surface. The bisaminomethylbithiazoles corrected $\triangle$ F508-CFTR in $\Delta$ F508/ $\Delta$ F508 human bronchial epithelia but did not correct a different temperature-sensitive CFTR mutant (P574H-CFTR) or a dopamine receptor mutant. Small-molecule correctors may be useful in the treatment of CF caused by the $\Delta$ F508 mutation.
\end{abstract}

\section{Introduction}

Cystic fibrosis (CF) is one of the most common inherited diseases, afflicting 1 in approximately 2,500 white individuals (1). The primary cause of morbidity and mortality in CF is chronic lung infection and deterioration of lung function. CF is caused by mutations in the CF transmembrane conductance regulator (CFTR) gene, which encodes a cAMP-regulated chloride channel expressed at the apical membrane of epithelial cells in the airways, pancreas, testis, and other tissues $(2,3)$. The most common CFTR mutation producing CF is deletion of phenylalanine at residue $508(\Delta \mathrm{F} 508)$ in its amino acid sequence, which is present in at least 1 allele in approximately $90 \%$ of CF subjects (1). The $\Delta$ F508-CFTR protein is misfolded and retained at the ER, where it is degraded rapidly (4-7). When allowed to traffic out of the ER by overexpression or by low-temperature incubation, $\Delta$ F508-CFTR also has a reduced half-life in the plasma membrane compared with wild-type $\operatorname{CFTR}(8,9)$. The misfolding of $\triangle$ F508-CFTR is thought to be mild because it can be "rescued" in cell culture models by incubation for 18 hours or more at reduced $\left(<30^{\circ} \mathrm{C}\right)$ temperature $(4)$ or with chemical chaperones such as glycerol (10) or phenylbutyrate (11), which results in partial restoration of $\Delta$ F508-CFTR plasma membrane expression. However, channel gating of the plasma membrane-rescued

Nonstandard abbreviations used: BHK, baby hamster kidney; $\mathrm{CF}$, cystic fibrosis; CFTR, CF transmembrane conductance regulator; DRD4, dopamine receptor 4; FRT, Fischer rat thyroid; $\Delta$ F508, deletion of phenylalanine at residue 508 ; $\Delta$ F508-CFTR$\mathrm{C}_{\mathrm{t}} \mathrm{HA}, \Delta \mathrm{F} 508$-CFTR with a C-terminal HA tag; 4-PBA, 4-phenylbutyrate; YFP, yellow fluorescent protein.

Conflict of interest: The authors have declared that no conflict of interest exists.

Citation for this article: J. Clin. Invest. 115:2564-2571 (2005).

doi:10.1172/JCI24898.
$\Delta$ F508-CFTR protein remains defective such that its open probability after cAMP stimulation is reduced by more than 3 -fold compared with that of wild-type CFTR $(12,13)$.

Small-molecule correctors of defective $\Delta$ F508-CFTR folding/ cellular processing ("correctors") and channel gating ("potentiators") may provide a strategy for therapy of CF that corrects the underlying defect. A potential advantage of pharmacotherapy for defective $\Delta$ F508- CFTR processing and gating is that it minimizes concerns about treating the wrong cells or losing physiological CFTR regulation, as might occur with gene therapy or activation of alternative chloride channels. Small-molecule $\Delta$ F508-CFTR potentiators with relatively low potency (>10-50 $\mu \mathrm{M})$ have been characterized, including the flavone genistein and the xanthine isobutylmethylxanthine (14-17). Recently, our laboratory identified by high-throughput screening tetrahydrobenzothiophene, phenylglycine, and sulfonamide potentiators that correct defective $\Delta$ F508-CFTR gating at concentrations below $100 \mathrm{nM}$ by a mechanism involving increased channel open time $(18,19)$. These potentiators were effective in human CF airway epithelial cells expressing $\Delta$ F508-CFTR and so may be useful in the therapy of CF caused by the $\Delta \mathrm{F} 508$ mutation when combined with a corrector of defective cellular processing or as monotherapy for a subset of patients having some $\Delta$ F508-CFTR plasma membrane expression.

Here, we report the identification and characterization of $\Delta F 508$ CFTR correctors by screening of a large collection of diverse small molecules. The discovery of useful correctors was anticipated to present a substantially greater challenge than the discovery of CFTR inhibitors $(20,21)$ or activators $(18,19,22)$ because protein folding and trafficking is a complex, multistep process involving multiple cellular targets, some of which may be cell type specific. 
A

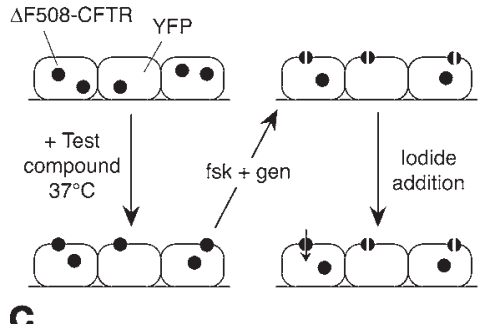

C

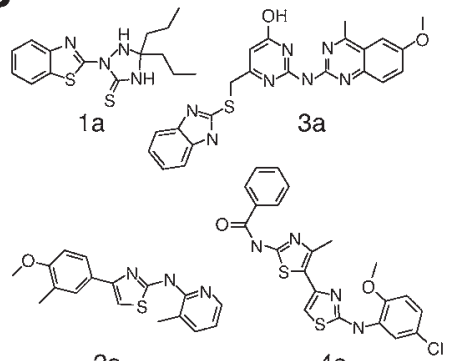

$2 a$

$4 a$

\section{Figure 1}

Discovery of $\triangle F 508-C F T R$ correctors by high-throughput screening. (A) Screening procedure. FRT cells coexpressing $\triangle$ F508-CFTR and a halide-sensitive YFP were incubated with test compounds $(10 \mu \mathrm{M})$ at $37^{\circ} \mathrm{C} . \Delta \mathrm{F} 508$-CFTR function was assayed at 18-24 hours in a plate reader according to YFP fluorescence quenching by iodide in the presence of forskolin $(20 \mu \mathrm{M})$ plus genistein (fsk + gen) $(50 \mu \mathrm{M})$. (B) Representative traces showing iodide influx under control conditions $\left(37^{\circ} \mathrm{C}\right)$ or after 24 hours incubation at $27^{\circ} \mathrm{C}$ or with 4-PBA $(4 \mathrm{mM})$ or correctors $(10 \mu \mathrm{M}$; $37^{\circ} \mathrm{C}$ ). (C) Chemical structures of correctors of 4 chemical classes. See Supplemental Table 1 for full listing of correctors and their activities. (D) Dose-response data for indicated correctors (SEM; $n=5$ ). The dashed line indicates the level of activity of the low-temperature rescue used as a positive control.

As depicted in Figure 1A, our screening assay utilized Fischer rat thyroid (FRT) epithelial cells coexpressing $\triangle$ F508-CFTR and the yellow fluorescent protein (YFP) halide indicator YFP-H148Q/ I152L at $37^{\circ} \mathrm{C}$ in a 96 -well-plate format. Test compounds at $10 \mu \mathrm{M}$ concentration (and negative/positive controls) were added to each well for 18-24 hours. $\Delta$ F508-CFTR-facilitated iodide influx was then determined from the kinetics of decreasing YFP fluorescence following addition of extracellular iodide in the presence of the potentiators genistein and forskolin. "Hits" from the primary high-throughput screen were verified, optimized by screening of structural analogs, and analyzed for mechanism of action and efficacy in human airway epithelia.

\section{Results}

Discovery and characterization of $\triangle F 508$-CFTR correctors. FRT cells coexpressing $\Delta$ F508-CFTR and a fluorescent YFP halide sensor were incubated with test compounds $(10 \mu \mathrm{M})$ for $18-24$ hours at $37^{\circ} \mathrm{C}$ in a 96 -well format (Figure $1 \mathrm{~A}$ ). $\Delta \mathrm{F} 508$-CFTR-facilitated iodide influx was then assayed after compound washout and addition of forskolin and genistein. Primary screening of 150,000 compounds produced 45 compounds that increased iodide influx $\left(\Delta \mathrm{d}\left[\mathrm{I}^{-}\right] / \mathrm{dt}\right)$ by more than $0.10 \mathrm{mM} / \mathrm{s}$ and $15 \mathrm{com}-$ pounds that increased iodide influx by more than $0.20 \mathrm{mM} / \mathrm{s}$. Examples of original data from individual wells are shown in Figure $1 \mathrm{~B}$. The negative control was vehicle (DMSO) alone (labeled " $37^{\circ} \mathrm{C}$ "), and the positive controls were 4-phenylbu- tyrate (4-PBA) and reduced-temperature $\left(27^{\circ} \mathrm{C}\right)$ incubation. Examples of 2 active compounds are shown. Active compounds were retested, and $\Delta$ F508-CFTR specificity was verified by inhibition of the increased iodide influx by a CFTR inhibitor $\left(\mathrm{CFTR}_{\text {inh }}-172\right)$ and lack of corrector effect on FRT-null cells.

Correctors were initially optimized by screening of commercially available analogs. Three rounds of optimization with more than 1,500 compounds gave active correctors of the aminobenzothiazole, aminoarylthiazole, quinazolinylaminopyrimidinone, and bisaminomethylbithiazole chemical classes (Figure 1C). Dose-response data are shown in Figure 1D, referenced against $27^{\circ} \mathrm{C}$ rescue, which is shown as the dashed line. A listing of 37 correctors and their potencies (acid ionization constant $\left[K_{\mathrm{a}}\right]$ and $\left.V_{\max }\right)$ is provided in Supplemental Table 1 (available online with this article; doi:10.1172/JCI24898DS1), establishing a structureactivity data set. Figure $2 \mathrm{~A}$ summarizes $V_{\max }$ data for several correctors, comparing them with positive and negative controls. Several compounds resulted in greater $V_{\text {max }}$ than did $27^{\circ} \mathrm{C}$ rescue, and additive effects of compounds and $27^{\circ} \mathrm{C}$ rescue were found.

Figure $2 \mathrm{~B}$ shows results of Ussing chamber experiments in which apical membrane chloride current was measured in FRT cells after basolateral membrane permeabilization and in the presence of a chloride gradient (apical, $65 \mathrm{mM}$; basolateral, $130 \mathrm{mM}$ ). After measurement of apical membrane chloride current at baseline, high concentrations of forskolin $(20 \mu \mathrm{M})$ and then genistein $(50 \mu \mathrm{M})$ were added; CFTR $_{\text {inh }}-172(10 \mu \mathrm{M})$ was added at the end of each experiment. The electrophysiological studies confirmed the data obtained from the fluorescence assay. On the left in Figure 2B is shown the much greater current in $\triangle \mathrm{F} 508$-CFTR-expressing cells grown at $27^{\circ} \mathrm{C}$ versus $37^{\circ} \mathrm{C}$ (top and middle curves) and the lack of corrector effect on FRT-null cells (bottom). Incubation of $\triangle \mathrm{F} 508$-CFTR-expressing cells with correctors at $37^{\circ} \mathrm{C}$ for 24 hours produced increased forskolin/genistein-stimulated and

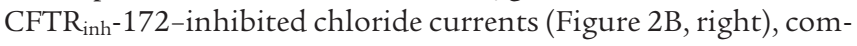
parable to or greater than those produced by $27^{\circ} \mathrm{C}$ rescue.

Figure $2 \mathrm{C}$ summarizes the time course of correction for 5 correctors (incubated at $37^{\circ} \mathrm{C}$ ), with data for $27^{\circ} \mathrm{C}$ rescue and 4-PBA shown for comparison. Correction was seen as early as 3 hours after compound addition, with maximal effect after 12-30 hours, whereas correction by $27^{\circ} \mathrm{C}$ incubation or 4-PBA had a relatively slower onset. Data for the persistence of correction after compound washout (or return of temperature from $27^{\circ} \mathrm{C}$ to $37^{\circ} \mathrm{C}$ ) are summarized in Figure 2D. Correction persisted beyond 12 hours for most compounds after washout, with substantial activity remaining for 2 of the correctors at 24 hours. In contrast, little correction persisted at 24 hours for cells rescued at $27^{\circ} \mathrm{C}$.

Experiments were performed to investigate whether the correctors might alter the properties of $\triangle F 508$-CFTR, such as the sensitivity to cAMP-elevating agents or to potentiators. Figure $3 \mathrm{~A}$ summarizes $V_{\max }$ in the presence of forskolin alone (at $20 \mu \mathrm{M}$ ) versus forskolin plus genistein $(50 \mu \mathrm{M})$. Interestingly, the fractional $V_{\max }$ produced by forskolin alone versus forskolin plus genistein was greater in cells treated with some correctors than that produced by low-temperature rescue. Thus, several correctors increased 

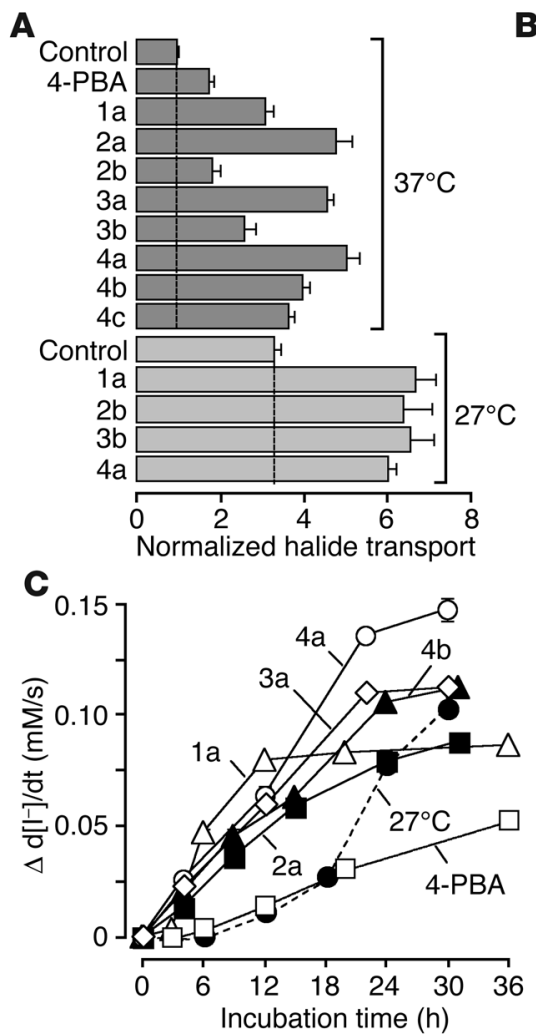

B
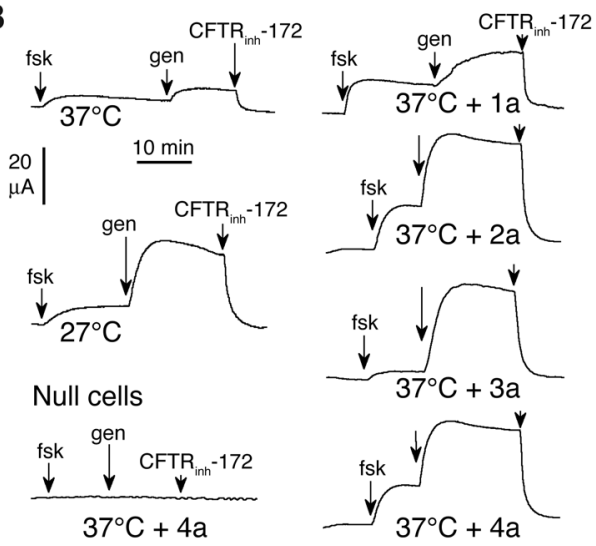

D

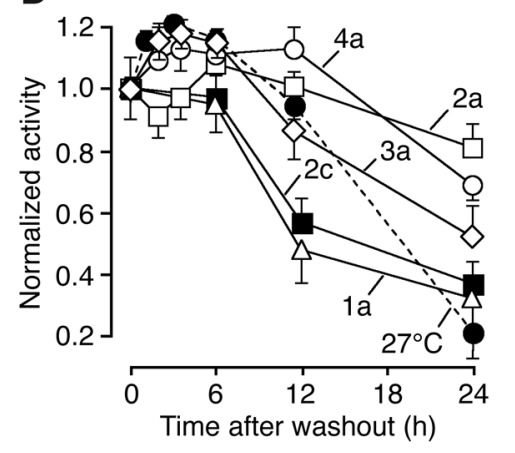

\section{Figure 2}

Properties of $\Delta$ F508-CFTR correctors. (A) Maximal iodide influx (normalized to $37^{\circ} \mathrm{C}$ control) in $\triangle$ F508-CFTR-expressing FRT cells incubated at $37^{\circ} \mathrm{C}$ or $27^{\circ} \mathrm{C}$ (SEM; $\left.n=5\right)$. lodide influx increased significantly $(P<0.01)$ for all compounds compared with control. (B) Apical membrane chloride current measured in Ussing chambers after basolateral membrane permeabilization and in the presence of a chloride gradient (see Methods). The concentrations were: forskolin, $20 \mu \mathrm{M}$; genistein, $50 \mu \mathrm{M}$; CFTR $_{\text {inh }}-172,10 \mu \mathrm{M}$. Measurements were performed on $\triangle$ F508-CFTR-expressing FRT cells, except in the experiment represented in the lower-left panel, which was performed on FRTnull cells. (C) Time course of correction. Cells incubated for different times at $27^{\circ} \mathrm{C}$ or with indicated correctors or 4-PBA $(4 \mathrm{mM})$ at $37^{\circ} \mathrm{C}$. $\triangle$ F508-CFTR activity was assayed in the presence of foskolin/genistein. (D) Persistence of correction. Cells were treated for 24 hours with correctors (or at $27^{\circ} \mathrm{C}$ ). $\Delta$ F508-CFTR activity was assayed at different times after washout (or return from $27^{\circ} \mathrm{C}$ to $37^{\circ} \mathrm{C}$ ).
$\Delta$ F508-CFTR activation by forskolin alone. Corrector $2 \mathrm{~b}$ (corr-2b) was most effective, with the forskolin response representing approximately $80 \%$ of $V_{\max }$. Figure $3 \mathrm{~B}$ shows dose-response data for forskolin (in the absence of genistein). Although $V_{\max }$ differed, $K_{\mathrm{a}}$ for the forskolin response was approximately $3 \mu \mathrm{M}$ in each case. As seen in Ussing chamber experiments (Figure 3C), corr-2b at $20 \mu \mathrm{M}$ (and to an even greater extent at $50 \mu \mathrm{M}$ ) increased the relative amplitude of the forskolin response. This was not due to intrinsic potentiator activity of corr- $2 \mathrm{~b}$, as it was unable to stimulate CFTR activity in cells rescued at $27^{\circ} \mathrm{C}$, with genistein as positive control (Figure 3C, far right).

Mechanism of action of $\triangle F 508$-CFTR correctors. Biosynthetic processing of $\triangle F 508$-CFTR by correctors was assayed by immunoblot analysis from the accumulation of mature, complex-glycosylated $\Delta$ F508-CFTR with a C-terminal HA tag $\left(\Delta\right.$ F508-CFTR- $\left.\mathrm{C}_{\mathrm{t}} \mathrm{HA}\right)$ in baby hamster kidney $(\mathrm{BHK})$ cells. Incubation of cells with correctors for $16-24$ hours at $37^{\circ} \mathrm{C}$ resulted in the accumulation of complex-glycosylated $\Delta$ F508-CFTR (Figure 4A). This was indicated by the slower electrophoretic mobility (apparent mol wt, approximately $170 \mathrm{kDa}$ ) of the complex-glycosylated $\Delta$ F508-CFTRimmunoreactive band ( $\mathrm{C}$ band) compared with its core-glycosylated counterpart (apparent mol wt, approximately $150 \mathrm{kDa}$ ), the predominant form in nontreated cells. Similar results were obtained in $\triangle$ F508-CFTR-expressing FRT cells (Figure 4B); however, the accumulation of $\mathrm{C}$ band was less prominent, which in part reflects the lower CFTR expression capacity of FRT cells (compare with wild-type CFTR in Figure 4, A and B). Interestingly, the accumulation of complex-glycosylated $\triangle$ F508-CFTR in corr-4-treated cells was comparable to or greater than that produced by low temperature incubation without correctors (Figure 4, A and B). Correctors did not produce an unfolded protein response (UPR) in BHK or FRT cells, as indicated by the unaltered expression of the UPR markers Grp78/Bip and GRP94 (23) (Figure 4B and data not shown). Plasma membrane $\Delta$ F508-CFTR expression was confirmed through use of an anti-HA antibody binding assay based on the recognition of an extracellular HA epitope (24). Incubation with correctors increased the abundance of plasma membrane $\Delta F 508$-CFTR significantly, with an approximately proportionate increase in forskolin/genistein-stimulated apical membrane chloride current measured in FRT cells (Figure 4C). Semiquantitative transport measurements in YFP-transfected BHK cells verified functional correction of $\triangle \mathrm{F} 508$-CFTR processing, with 2.5-, 5.2-, 2.6-, and 5.9-fold increases in $\mathrm{I}^{-}$influx in cells treated with correctors $1 \mathrm{a}$, 2a, 3a, and $4 \mathrm{a}$, respectively, compared with cells treated with vehicle (DMSO) alone (data not shown).

The $\Delta$ F508 mutation impairs $\Delta F 508$-CFTR conformational maturation and export competence at the ER and destabilizes complex-glycosylated $\Delta$ F508-CFTR in post-Golgi compartments $(6,8,24)$. Correctors may facilitate translation or posttranslational folding of newly synthesized $\Delta$ F508-CFTR and/or enhance the stability of mature, complex-glycosylated $\Delta \mathrm{F} 508$ CFTR. We examined these possibilities to establish the cellular basis of corrector action.

First, we determined whether the correctors augmented $\Delta \mathrm{F} 508$ CFTR transcription and/or translation. Figure 4D shows that the correctors did not alter the amount of newly synthesized $\Delta$ F508CFTR as measured by short metabolic pulse labeling, which provides evidence against altered $\Delta F 508$-CFTR transcription/translation. Next, corrector effect on $\Delta$ F508-CFTR posttranslational folding efficiency was assessed by measurement of the fractional conversion of newly synthesized, core-glycosylated $\Delta$ F508-CFTR 

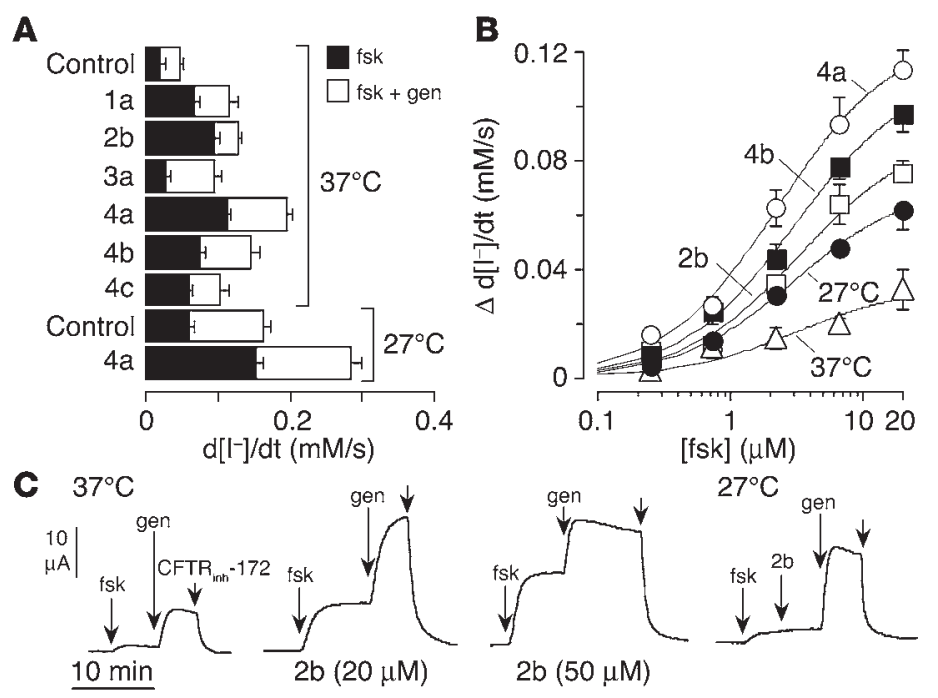

\section{Figure 3}

Increased $\triangle F 508$-CFTR sensitivity to activators after incubation with correctors. (A) Effect of forskolin $(20 \mu \mathrm{M})$ or forskolin plus genistein $(50 \mu \mathrm{M})$ in $\Delta \mathrm{F} 508$-CFTR-expressing FRT cells kept at $37^{\circ} \mathrm{C}$ or $27^{\circ} \mathrm{C}$ with or without correctors. (B) Forskolin doseresponse relationships. Cells stimulated with forskolin (in the absence of genistein) after incubation with correctors (at $37^{\circ} \mathrm{C}$ ) or at $27^{\circ} \mathrm{C}$. (C) Apical membrane chloride current after incubation for 24 hours at $37^{\circ} \mathrm{C}$ with DMSO vehicle (left curve) or corr-2b (middle curves). The curve at the far right was obtained from an experiment on cells grown at $27^{\circ} \mathrm{C}$ with compounds added as shown ( $20 \mu \mathrm{M}$ forskolin, $20 \mu \mathrm{M}$ corr-2b, $50 \mu \mathrm{M}$ genistein). into the complex-glycosylated form (Figure 4E, top). When the radioactive pulse labeling was extended from 15 to 150 minutes, the detection sensitivity of the assay was enhanced enough to allow folding efficiency to be estimated. Phosphorimage analysis showed that $\triangle$ F508-CFTR had low but measurable maturation efficiency $(0.5 \% \pm 0.15 \%)$ as compared with wild-type CFTR $(31 \% \pm 5 \%)$ in BHK cells at $37^{\circ} \mathrm{C}$ (Figure 4E, bottom). Similar results have been obtained for maturation efficiency of wild-type CFTR in other heterologous expression systems $(5,25)$. $\triangle F 508$-CFTR folding efficiency was increased by 2 - to 3 -fold by class 4 correctors (Figure 4E). Class 4 correctors increased the folding efficiency of wild-type CFTR by 5-70\% (data not shown).

Efficient correctors may function as pharmacological chaperones by improving $\Delta$ F508-CFTR posttranslational folding and stabilizing its ER core-glycosylated form. We found that class 4 correctors delayed by 30-50\% ER-associated degradation of metabolically labeled core-glycosylated $\Delta$ F508-CFTR (Figure 4F).

Slowing of the normally rapid elimination of $\triangle F 508$-CFTR from the cell surface may also contribute to its functional rescue. For assessment of the plasma membrane stability of rescued $\Delta$ F508CFTR, $\triangle$ F508-CFTR with a 3 -HA tag ( $\Delta$ F508-CFTR-3HA) was accumulated at the cell surface at reduced temperature. $\Delta \mathrm{F} 508$ CFTR surface density was then monitored by antibody binding after the temperature was returned to $37^{\circ} \mathrm{C}$ in the presence of correctors. Whereas the cell-surface density of rescued $\Delta$ F508-CFTR was reduced to approximately $35 \%$ during a 3 -hour chase, $50-60 \%$ of the $\Delta$ F508-CFTR remained at the cell surface in the presence of correctors 3a, 4a, and 4b (Figure 4G). Intriguingly, corr-2a was the most potent stabilizer, though it had no effect on $\triangle$ F508-CFTR folding in the ER, which suggests that the primary target of corr$2 \mathrm{a}$ is the peripheral quality control machinery that is responsible for targeting misfolded $\Delta$ F508-CFTR for lysosomal degradation (24). These results also indicate that class 4 correctors not only improve $\Delta$ F508-CFTR folding, but also enhance the residence time of rescued $\Delta F 508$-CFTR at the cell surface.

As an initial test of compound specificity for correction of defective $\Delta F 508$-CFTR processing, compounds were tested on $\mathrm{P} 574 \mathrm{H}$-CFTR, a mutant that like $\Delta \mathrm{F} 508$-CFTR is retained at the ER but can be rescued by incubation for 24 hours at reduced temperature (26). Cells were incubated with correctors or at reduced temperature for 24 hours. Apical membrane chloride current was measured in response to forskolin (which fully activates this CFTR mutant; ref. 27) and then $\mathrm{CFTR}_{\text {inh }}-172$. Figure $5 \mathrm{~A}$ shows that corr $-4 \mathrm{a}$ and $-2 \mathrm{a}$, which produced robust chloride currents in $\triangle F 508$-CFTR-expressing cells, did not produce significant correction in P574H-CFTR cells, despite a positive low-temperature rescue control. Corr-1a and - 3 a caused a small but significant increase in CFTR-dependent current (Figure 5A). We further substantiated corrector specificity using a mutant $G$ protein-coupled receptor that is misfolded and retained in the ER (28). While the processing defect of a mutant dopamine receptor 4 (DRD4) was efficiently rescued by the pharmacological chaperone domperidone as assayed by cell surface antibody binding measurements, class 4 compounds did not correct defective DRD4 processing (Figure 5B).

Correction of defective $\triangle F 508$-CFTR processing in human airway epithelium. Last, functional measurements of corrector action were performed on well-differentiated primary cultures of human bronchial epithelial cells from $\Delta$ F508-CFTR-homozygous subjects. Cells polarized on permeable supports were mounted in Ussing chambers for measurement of chloride secretion by short-circuit current analysis. After $\mathrm{Na}^{+}$current was blocked with amiloride, cells treated with DMSO vehicle alone showed little response to forskolin, genistein, or $\mathrm{CFTR}_{\text {inh }}-172$ (Figure 6A, top panel). Incubation at $27^{\circ} \mathrm{C}$ for 24 hours resulted in the appearance of significant chloride current as indicated by the increased current after addition of forskolin and genistein and inhibition by $\mathrm{CFTR}_{\text {inh- }}-172$ (Figure $6 \mathrm{~A}$, middle panels). Incubation with corr- 4 a at $37^{\circ} \mathrm{C}$ for 24 hours increased chloride current comparably. For comparison, data for non-CF bronchial epithelial cells are shown (Figure 6A, bottom panel). Figure 6B summarizes the $\mathrm{CFTR}_{\text {inh }}-172$-sensitive short-circuit for the measurements used in Figure 6A, including data for a second bisaminomethylbithiazole. Corr-4a produced a significant increase in the CFTR-dependent $\mathrm{Cl}^{-}$secretion, which was equivalent to $8 \%$ of that measured in non-CF epithelia (Figure 6B). The other compounds tested (corr-1a, $-2 \mathrm{a}$, and -3a) did not significantly increase CFTR-dependent $\mathrm{Cl}^{-}$secretion (data not shown). As a negative control for these studies, the active correctors were tested on human bronchial epithelial cells derived from a CF 
A

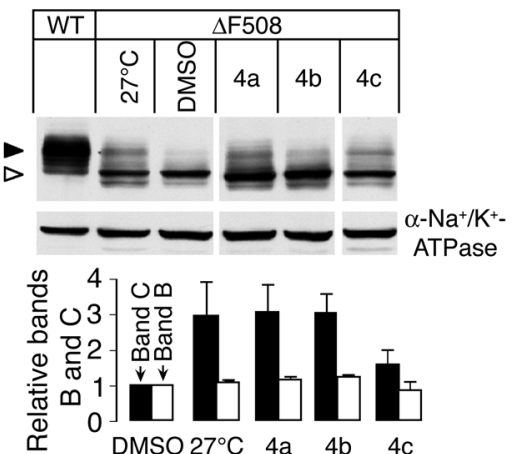

B

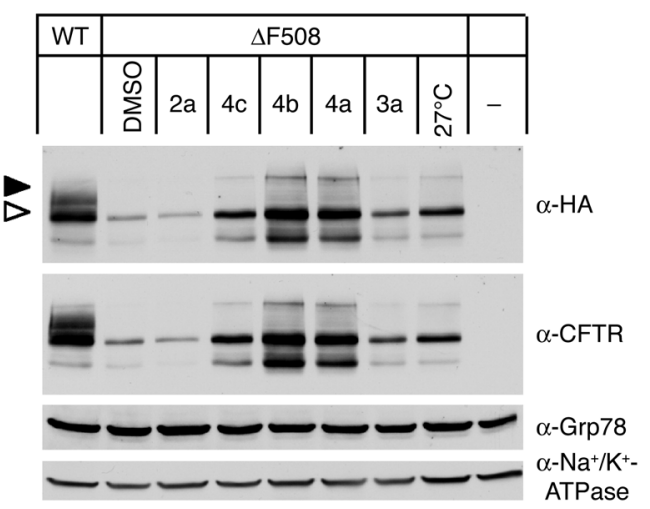

E

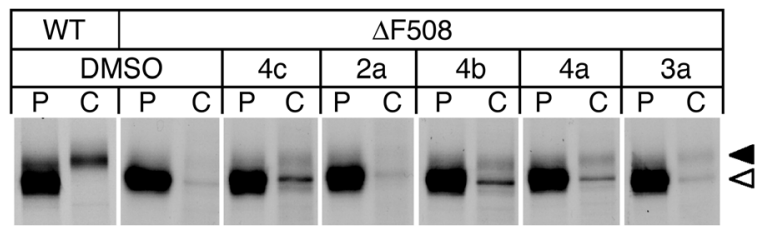

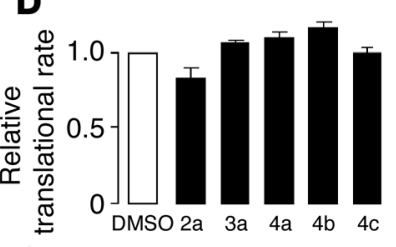

G

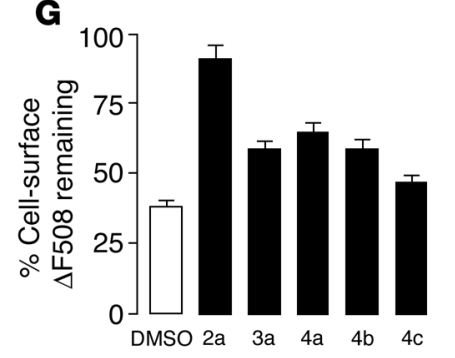

C

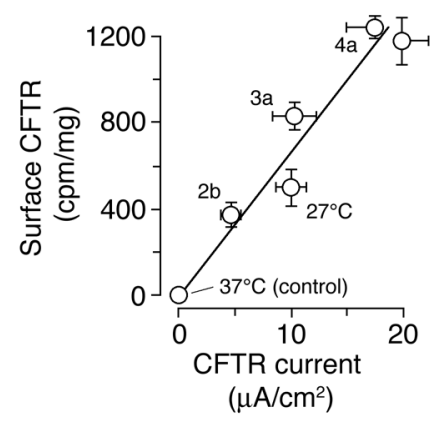

$\mathbf{F}$

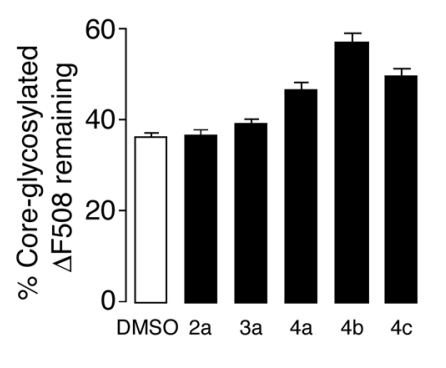

Figure 4

Biochemical analysis of corrector mechanism of action. (A) Effect of the indicated correctors (10 $\mu \mathrm{M})$ on the expression pattern of $\Delta \mathrm{F} 508-\mathrm{CFTR}-\mathrm{C}_{\mathrm{t}} \mathrm{HA}$ in BHK cells. Cells were cultured for 24 hours at $37^{\circ} \mathrm{C}$ with or without correctors or at $27^{\circ} \mathrm{C}$. Top: CFTR was visualized using anti-HA primary and HRP-conjugated secondary antibodies. Filled arrowhead, complex-glycosylated form (band C); open arrowhead, core-glycosylated form (band B). Bottom: Quantification of bands B and C (SEM; $n=4-5)$. (B) Effect of the indicated correctors (10 $\mu M)$ on the expression pattern of $\Delta$ F508-CFTR in FRT epithelia. Confluent monolayers were treated as described in A, and immunoblot analysis was performed with the indicated primary antibodies. (C) Correlation between cell-surface density and PKA-activated chloride current. Cell-surface density of $\Delta$ F508-CFTR was determined using the radioactive anti-HA antibody binding assay and plotted against $\triangle F 508-C F T R$ apical membrane currents in parallel experiments done on FRT cells. (D) Translational rate of $\Delta$ F508-CFTR was measured in the presence of correctors (10 $\mu \mathrm{M})$ by monitoring [ ${ }^{35}$ S]methionine/cysteine incorporation into CFTR during a 15-minute pulse labeling (SEM; $n=3)$. (E) Folding efficiency measured by pulse-chase assay. Top: The amount of newly synthesized $\Delta$ F508-CFTR was computed from radioactive incorporation during a 15-minute pulse $(P)$. For measurement of folding efficiency, cells were pulsed for 150 minutes and than chased for 150 minutes (C). The amounts of core- (open arrowhead) and complex-glycosylated (filled arrowhead) forms were determined by phosphorimage analysis. Bottom: Maturation efficiency expressed as the percent of mature, complex-glycosylated $\Delta \mathrm{F} 508-$ CFTR relative to the newly synthesized pool, as shown in the top panel. (F) Stability of the core-glycosylated $\Delta$ F508-CFTR was measured following a 15-minute pulse labeling in the presence of the indicated correctors. Remaining radioactivity associated with CFTR was measured after 1 hour chase (SEM; $n=3)$. (G) Cell-surface stability of the rescued $\Delta$ F508-CFTR was measured by the anti-HA antibody binding assay before and after 2 hours chase in the presence of the indicated correctors. Data are expressed as the mean \pm SEM of 3 independent experiments.

subject homozygous for the N1303K-CFTR mutation, which also manifests defective CFTR processing (29). Figure 6C shows that there was no significant correction of short-circuit current for the N1303K-CFTR bronchial cells when measured with the same compounds and conditions used for the $\Delta$ F508-CFTR cells measured in Figure 6A.

\section{Discussion}

High-throughput screening yielded several classes of small molecules that corrected $\Delta F 508$-CFTR cellular misprocessing and restored plasma membrane expression and halide permeability to levels greater than those achieved by low-temperature rescue. We verified correction by electrophysiological and biochemical measurements, as well as using control (non- $\Delta$ F508-CFTRexpressing) cells and a CFTR-selective inhibitor. The micromolar potency of the correctors is orders of magnitude better than the millimolar potency reported for correction of $\triangle F 508$-CFTR misprocessing by 4-PBA (11). The identification of small-molecule $\Delta F 508$-CFTR correctors presented a greater conceptual difficulty than that of $\Delta$ F508-CFTR potentiators or CFTR activators/inhibitors because correction of cellular misprocessing could involve multiple targets, whereas the primary target for potentiators, activators, and inhibitors is CFTR itself.

The cell line used for primary screening was chosen to be of epithelial origin (to resemble native CFTR-expressing cells), to permit rapid assessment of chloride currents in cell monolayers, 
A
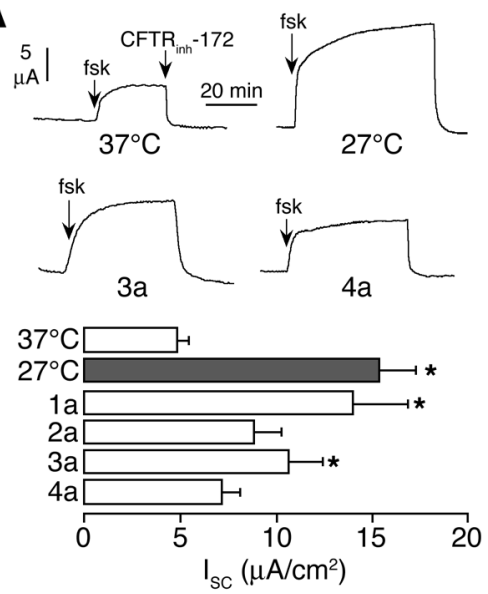

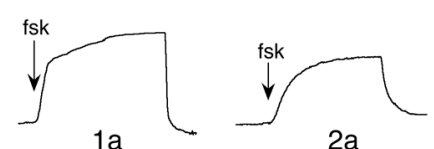

$2 a$

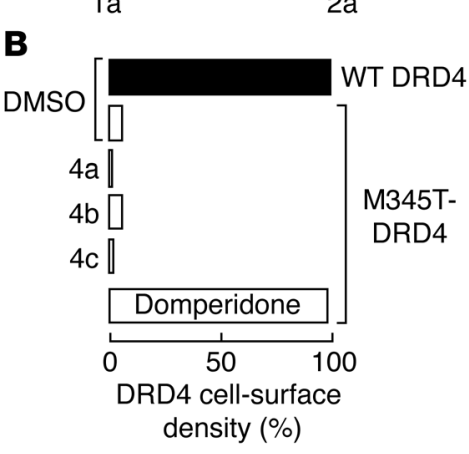

Figure 5

Specificity for corrector action on $\triangle F 508-C F T R$ cellular misprocessing. (A) Apical membrane chloride current in FRT cells expressing the temperature-sensitive mutant P574H-CFTR. Experiments were carried out as described in Figure 2B. Isc, short-circuit current. (B) $\mathrm{CHO}$ cells expressing Flag-tagged wild-type or mutant (M345T) dopamine receptor (DRD4) were treated for 16 hours with the indicated correctors ( $5 \mu \mathrm{M}$ corr- $4 \mathrm{c}$ and $-4 \mathrm{~b} ; 10 \mu \mathrm{M}$ corr- $4 \mathrm{a})$ or $10 \mu \mathrm{M}$ domperidone, a dopamine antagonist. The cellsurface density of DRD4 was measured using anti-Flag primary and HRP-conjugated goat anti-mouse antibodies. Data represent the mean of 2 independent experiments, each performed in triplicate. and to give a robust low-temperature rescue response. Additional requirements for the cell line for high-throughput screening included rapid growth in multiwell plates, stable and bright YFP-H148Q/I152L expression, and low basal halide permeability. The YFP-H148Q/I152L fluorescent halide indicator was developed by our lab (30) to have bright cellular expression and ultra-high iodide sensitivity. The transfected FRT cell line used here was selected after many transfected and natively expressed epithelial cell lines were screened, and well as more than $100 \Delta \mathrm{F} 508$-CFTR/ YFP-H148Q/I152L-transfected FRT cell clones (18). Although primary screening using natively expressed human airway cells may be preferable on theoretical grounds, practical considerations of stable cell phenotype, relatively high $\Delta$ F508-CFTR expression, and robust signal $\left(Z^{\prime}\right.$ factor $\left.>0.5\right)$ precluded the use of available human $\triangle$ F508-CFTR cell lines.

CFTR cellular processing involves translation, folding at the ER, Golgi transport, posttranslational glycosylation, and apical plasma membrane targeting (6). Plasma membrane CFTR is internalized by endocytosis and then recycled to the plasma membrane or targeted for lysosomal degradation $(9,24)$. $\Delta$ F508-CFTR folding is inefficient, with $99.5 \%$ of newly synthesized $\Delta$ F508-CFTR in BHK cells targeted for degradation without reaching the Golgi apparatus. Near-complete ER retention of $\triangle F 508$-CFTR was reported in other model systems as well $(2,6)$.

Screening of 150,000 chemically diverse compounds produced several classes of candidate correctors of defective $\Delta$ F508-CFTR cellular processing. Functional and biochemical analysis identified the class 4 bisami-

\section{Figure 6}

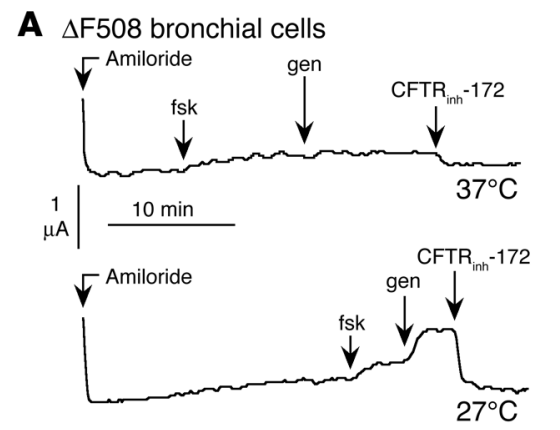

nomethylbithiazoles as the most promising class of compounds for further development. Corr-4a and $-4 \mathrm{c}$ increased $\triangle F 508-C F T R$ folding efficiency nearly 3 -fold without affecting translational rate, which suggests that the correctors could partially overcome the posttranslational folding barrier. Based on recent structural studies, small molecules might facilitate the folding of the nucleotide binding domain 2 (NBD2) and/or transmembrane domains $(7,31)$. The simplest interpretation of the peripheral stabilizing

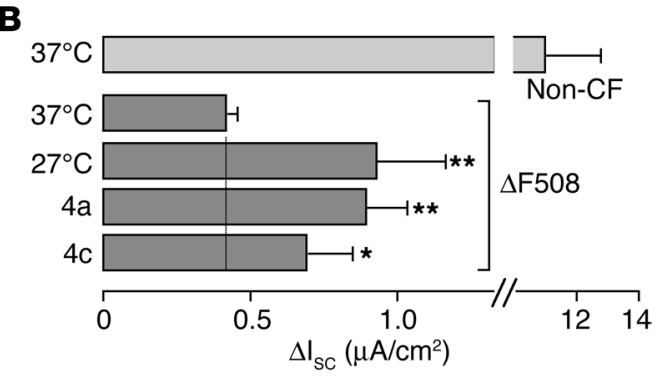

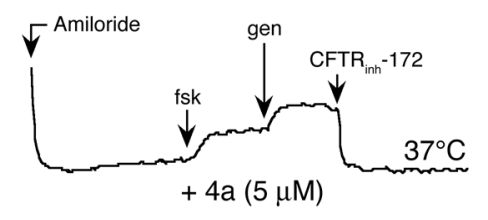
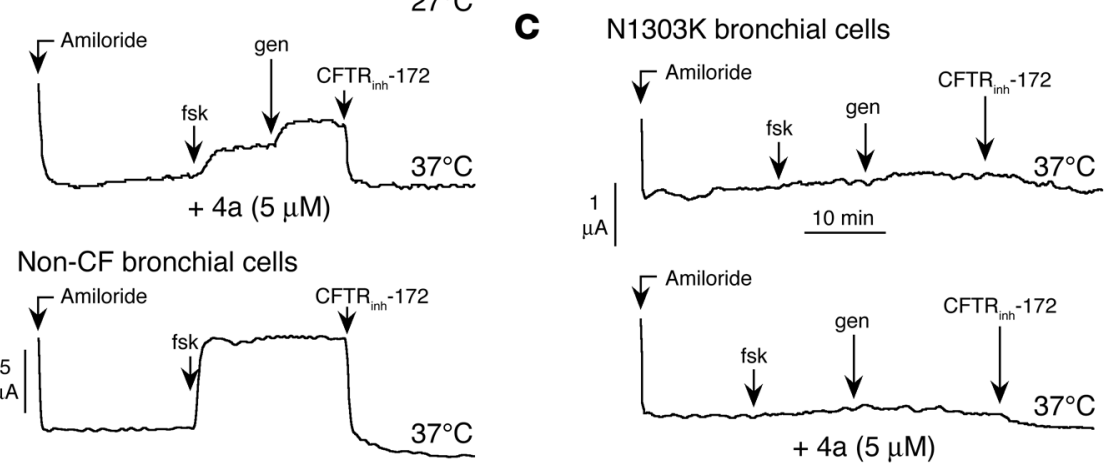

Correction of $\Delta F 508-C F T R$ misprocessing in human bronchial epithelial cells. (A) Representative short-circuit current recordings on primary cultures of human bronchial epithelial cells from a $\Delta F 508 /$ $\Delta \mathrm{F} 508$ subject (top 3 curves) and a non-CF subject (bottom curve). $\Delta \mathrm{F} 508$ cells maintained at $37^{\circ} \mathrm{C}$ for 24 hours in the presence of DMSO vehicle (top curve) or corr-4a (third curve) or incubated at $27^{\circ} \mathrm{C}$ (second curve). Concentrations were: amiloride, $10 \mu \mathrm{M}$; forskolin, $20 \mu \mathrm{M}$; genistein, $50 \mu \mathrm{M}$; CFTR $_{\text {inh }}-172,10 \mu \mathrm{M}$. (B) Summary of CFTR inh $-172-$ inhibited short-circuit current $\left(\Delta \mathrm{I}_{\mathrm{sc}}\right)$ for a series of experiments as described in A (SEM; $n=12-14)$. ${ }^{*} P<0.05 ;{ }^{* *} P<0.01$. (C) Short-circuit current recordings of primary cultures of human bronchial epithelial cells from a homozygous N1303KCFTR subject taken under conditions as described in $\mathbf{A}$. 
effect is that the conformationally stabilized mutant is less susceptible to the ubiquitin-dependent peripheral quality control mechanism and lysosomal degradation than the low temperature-rescued $\Delta$ F508-CFTR (24). The lack of effect of some $\Delta$ F508-CFTR correctors on the temperature-sensitive mutant P574H-CFTR and the mutant DRD4-M345T suggests their specificity for the $\Delta \mathrm{F} 508$ mutation, though further studies are needed to evaluate the numerous potential off-target specificities.

Our results thus provide proof-of-principle for discovery of small-molecule correctors of $\Delta \mathrm{F} 508$-CFTR cellular misprocessing. While some correctors, such as those of class 4 , have favorable properties, including efficacy in human airway epithelia, partial correction of the gating defect, apparent $\Delta$ F508-CFTR specificity, and a mechanism involving improved $\Delta$ F508-CFTR protein folding, additional analysis and compound optimization are needed prior to the initiation of clinical studies, to include in vivo testing of compound pharmacology, toxicity, and efficacy. The effect of class 4 correctors on $\mathrm{Cl}^{-}$secretion in human airway CF epithelial cells was small (approximately 8\%) compared with that in non-CF epithelia. However, the correction conferred by class 4 compounds is comparable to that obtained by low-temperature rescue and within the range considered to be therapeutically beneficial. It has been estimated that $6-10 \%$ of normal CFTR activity might prevent or significantly reduce lung pathology in CF (32), although this remains the subject of debate. Finally, the general paradigm of small molecule discovery of correctors of mutant protein misfolding may be applicable to a wide range of protein folding diseases such as Alzheimer disease, Parkinson disease, nephrogenic diabetes insipidus, and $\alpha 1$-antitrypsin deficiency $(33,34)$.

\section{Methods}

Cell lines. FRT epithelial cells stably coexpressing human $\triangle$ F508-CFTR and the high-sensitivity halide-sensing green fluorescent protein YFPH148Q/I152L (30) were generated as described previously $(22,35)$. A similar approach was used to generate $\mathrm{BHK}$ cells coexpressing $\Delta \mathrm{F} 508$ CFTR and YFP-H148Q/I152L and FRT cells stably expressing P574HCFTR. FRT cells were cultured on plastic in Coon's modified Ham's F12 medium supplemented with $10 \% \mathrm{FBS}, 2 \mathrm{mM}$ L-glutamine, $100 \mathrm{U} / \mathrm{ml}$ penicillin, and $100 \mu \mathrm{g} / \mathrm{ml}$ streptomycin. Generation of BHK cells expressing wild-type CFTR or $\triangle$ F508-CFTR- $\mathrm{C}_{\mathrm{t}} \mathrm{HA}$ (8). The extracellular triple HA (3HA) tag, consisting of SLEYPYDVPDYASYPYDVPDYAYPYDVPDYAA residues, was inserted after amino acid residue 897 in wild-type CFTR and $\triangle$ F508-CFTR using engineered XhoI/NheI sites. For primary screening, FRT cells were plated using a LabSystems multidrop dispenser into black, 96-well microplates (Costar; Corning Inc.) at 50,000 cells/well. Screening was done at 18-24 hours after plating. For short-circuit current measurements, cells were cultured on Snapwell permeable supports (Costar; Corning Inc.) at 500,000 cells/insert. Human nasal epithelial cells, which were obtained from $\Delta$ F508/ $\Delta$ F508 CF subjects (or an N1303K/N1303K subject) with informed consent, were cultured on Snapwell inserts and allowed to differentiate in a hormone-supplemented medium as described previously (36). CHO cells expressing the Flag-tagged wild-type and mutant DRD4 (28) were kindly provided by H.H.M. Van Tol (University of Toronto).

Compounds. A total of 150,000 diverse drug-like compounds (>90\% with a molecular size of 250-500 Da; ChemDiv Inc. and ChemBridge Corp.) was used for initial screening. For optimization, more than 1,500 commercially available analogs of active compounds identified in the primary screen were tested. Plates containing 1 or 4 compounds per well were prepared for screening (1 mM in DMSO). Compounds for secondary analysis were confirmed by NMR and liquid chromatography/mass spectrometry.

Screening procedures. Screening was carried out using a Beckman Coulter integrated system containing a 3-m robotic arm, $\mathrm{CO}_{2}$ incubator containing microplate carousel, plate-washer, liquid handling work station, bar code reader, delidding station, plate sealer, and 2 FLUOstar fluorescence plate readers (Optima; BMG LABTECH Gmbh), each equipped with dual syringe pumps and $500 \pm 10 \mathrm{~nm}$ excitation and $535 \pm 15 \mathrm{~nm}$ emission filters (Chroma Technology Corp.). FRT cells were grown at $37^{\circ} \mathrm{C}\left(90 \%\right.$ humidity; $5 \% \mathrm{CO}_{2}$ ) for $18-24$ hours and then incubated for $18-24$ hours with $50 \mu \mathrm{l}$ of medium containing test compounds $(10-\mu \mathrm{M}$ final concentrations). At the time of the assay, cells were washed with PBS and then incubated with PBS containing forskolin $(20 \mu \mathrm{M})$ and genistein $(50 \mu \mathrm{M})$. Each well was assayed individually for $\mathrm{I}^{-}$influx in a plate reader by recording fluorescence continuously ( $200 \mathrm{~ms}$ per point) for 2 seconds (baseline) and then for 12 seconds after rapid ( $<1$ second) addition of $165 \mu \mathrm{l}$ PBS in which $137 \mathrm{mM} \mathrm{Cl}^{-}$was replaced by $\mathrm{I}^{-}$. The $\mathrm{I}^{-}$influx rate was computed by fitting the final 11.5 seconds of the data to an exponential for extrapolation of initial slope and normalizing for background-subtracted initial fluorescence. All compound plates contained negative controls (DMSO vehicle) and positive controls (4-PBA, $4 \mathrm{mM}$ ), and separate positive control plates were used for low-temperature rescue $\left(27^{\circ} \mathrm{C}\right.$ incubation for $18-24$ hours). Assay analysis indicated a $Z^{\prime}$ factor (37) of more than 0.6.

Short-circuit current measurements. $\Delta$ F508-CFTR-expressing FRT cells were cultured on Snapwell inserts for 7-9 days. Test compounds were added 18-24 hours prior to measurements. The basolateral solution contained $130 \mathrm{mM}$ $\mathrm{NaCl}, 2.7 \mathrm{mM} \mathrm{KCl}, 1.5 \mathrm{mM} \mathrm{KH}_{2} \mathrm{PO}_{4}, 1 \mathrm{mM} \mathrm{CaCl}_{2}, 0.5 \mathrm{mM} \mathrm{MgCl}_{2}, 10 \mathrm{mM}$ glucose, and $10 \mathrm{mM} \mathrm{Na}$-HEPES (pH 7.3). In the apical bathing solution, $65 \mathrm{mM}$ $\mathrm{NaCl}$ was replaced by $\mathrm{Na}$ gluconate, and $\mathrm{CaCl}_{2}$ was increased to $2 \mathrm{mM}$. Solutions were bubbled with air and maintained at $37^{\circ} \mathrm{C}$. The basolateral membrane was permeabilized with $250 \mu \mathrm{g} / \mathrm{ml}$ amphotericin B. For studies of human bronchial epithelial cells, apical and basolateral chambers contained identical solutions: $126 \mathrm{mM} \mathrm{NaCl}, 0.38 \mathrm{mM} \mathrm{KH}_{2} \mathrm{PO}_{4}, 2.1 \mathrm{mM}$ $\mathrm{K}_{2} \mathrm{HPO}_{4}, 1 \mathrm{mM} \mathrm{MgSO}_{4}, 1 \mathrm{mM} \mathrm{CaCl}_{2}, 24 \mathrm{mM} \mathrm{NaHCO}_{3}$ and $10 \mathrm{mM}$ glucose (the basolateral membrane was not permeabilized). Hemichambers were connected to a DVC-1000 voltage clamp (World Precision Instruments Inc.) via $\mathrm{Ag} / \mathrm{AgCl}$ electrodes and $1 \mathrm{M} \mathrm{KCl}$ agar bridges for recording of apical membrane or short-circuit current.

Analysis of corrector mechanisms. Some biochemical studies were done on BHK cells stably transfected with HA-tagged wild-type CFTR or $\triangle$ F508CFTR. CFTR was tagged at the C-terminal tail (CFTR- $\mathrm{C}_{\mathrm{t}} \mathrm{HA}$ ) or in its fourth extracellular loop with 3 HA epitopes (CFTR-3HA) $(8,24)$. Accumulation of complex-glycosylated CFTR was assayed by immunoblot analysis as described previously (8). Plasma membrane expression was assayed by HA antibody binding (in nonpermeabilized cells) using an iodinated secondary anti-mouse antibody as described previously (24) or an HRP-coupled secondary antibody with Amplex Red (Invitrogen Corp.) as substrate. Nonspecific antibody binding was measured in nontransfected cells (using the same assay conditions) and in transfected cells with primary antibody omitted. The translational rate of $\triangle \mathrm{F} 508$-CFTR was measured as described previously (7). Folding efficiency was assayed as described previously (7) with modifications. $\Delta$ F508-CFTR- ${ }_{\mathrm{t}} \mathrm{HA}$-expressing cells were depleted of endogenous methionine and cysteine in the presence of correctors and then incubated with $0.2 \mathrm{mCi} / \mathrm{ml}\left[{ }^{35} \mathrm{~S}\right]$ methionine and $\left[{ }^{35} \mathrm{~S}\right]$ cysteine for 150 minutes and chased in culture medium for an additional 150 minutes. The amount of newly synthesized $\Delta$ F508-CFTR during 150 minutes of labeling was computed from radioactive incorporation during a 15-minute pulse, performed in parallel measurement. Radioactively labeled CFTR was isolated by immunoprecipitation, visualized by fluorography, and quantified by 
phosphorimage analysis as described previously (5). Maturation efficiency was expressed as the percentage of radioactivity associated with the complex-glycosylated form relative to the total amount of newly synthesized $\Delta$ F508-CFTR. Considering the metabolic stability of the complex-glycosylated $\triangle \mathrm{F} 508$-CFTR and its kinetics of biogenesis, we estimated that approximately $35 \%$ of the complex-glycosylated $\triangle$ F508-CFTR is degraded during the chase. Thus, the calculated maturation efficiencies in the absence of peripheral stabilization are slight underestimates.

\section{Acknowledgments}

We thank R. Kip Guy and A. Shelat for computational analysis in selecting compounds for primary screening and structure-activity analysis and Hubert H.M. Van Tol for providing the DRD4 express- ing cell lines. This work was supported by the Cystic Fibrosis Foundation and by grants DK72517, HL73856, HL59198, EB00415, EY13574, and DK35124 from the NIH. L. Galietta acknowledges grant GP0296Y01 from Telethon-Italy. G. Lukacs was supported in part by the Canadian Institutes of Health Research.

Received for publication March 1, 2005, and accepted in revised form June 21, 2005.

Address correspondence to: Alan S. Verkman, 1246 Health Sciences East Tower, University of California, San Francisco, San Francisco, California 94143-0521, USA. Phone: (415) 476-8530; Fax: (415) 665-3847; E-mail: verkman@itsa.ucsf.edu.
1. Bobadilla, J.L., Macek, M., Jr., Fine, J.P., and Farrell, P.M. 2002. Cystic fibrosis: a worldwide analysis of CFTR mutations - correlation with incidence data and application to screening [review]. Hum. Mutat. 19:575-606.

2. Pilewski, J.M., and Frizzell, R.A. Role of CFTR in airway disease. 1999. Physiol. Rev. 79:S215-S255.

3. Sheppard, D.N., and Welsh, M.J. 1999. Structure and function of the CFTR chloride channel. Physiol. Rev. 79:S23-S45.

4. Denning, G.M., et al. 1992. Processing of mutant cystic fibrosis transmembrane conductance regulator is temperature-sensitive. Nature. 358:761-764.

5. Lukacs, G.L., et al. 1994. Conformational maturation of CFTR but not its mutant counterpart ( $\triangle \mathrm{F} 508)$ occurs in the endoplasmic reticulum and requires ATP. EMBOJ. 13:6076-6086.

6. Kopito, R.R. 1999. Biosynthesis and degradation of CFTR. Physiol. Rev. 79:S167-S173.

7. Du, K., Sharma, M., and Lukacs, G.L. 2005. The $\Delta$ F508 cystic fibrosis mutation impairs domaindomain interactions and arrests post-translational folding of CFTR. Nat. Struct. Mol. Biol. 12:17-25.

8. Sharma, M., Benharouga, M., Hu, W., and Lukacs, G.L. 2001. Conformational and temperature-sensitive stability defects of the $\Delta \mathrm{F} 508$ cystic fibrosis transmembrane conductance regulator in postendoplasmic reticulum compartments. J. Biol. Chem. 276:8942-8950.

9. Gentzsch, M., et al. 2004. Endocytic trafficking routes of wild type and $\Delta \mathrm{F} 508$ cystic fibrosis transmembrane conductance regulator. Mol. Biol. Cell. 5:2684-2696.

10. Sato, S., Ward, C.L., Krouse, M.E., Wine, J.J., and Kopito, R.R. 1996. Glycerol reverses the misfolding phenotype of the most common cystic fibrosis mutation. J. Biol. Chem. 271:635-658.

11. Rubenstein, R.C., Egan, M.E., and Zeitlin, P.L. 1997. In vitro pharmacologic restoration of CFTR-mediated chloride transport with sodium 4-phenylbutyrate in cystic fibrosis epithelial cells containing AF508-CFTR. J. Clin. Invest. 100:2457-2465.

12. Dalemans, W., et al. 1991. Altered chloride ion channel kinetics associated with the $\Delta$ F508 cystic fibrosis mutation. Nature. 354:526-528.

13. Haws, C.M., et al. 1996. $\Delta$ F508-CFTR channels: kinetics, activation by forskolin, and potentiation by xanthines. Am. J. Physiol. 270:C1544-C1555.
14. Drumm, M.L., et al. 1991. Chloride conductance expressed by $\Delta \mathrm{F} 508$ and other mutant CFTRs in Xenopus oocytes. Science. 254:1797-1799.

15. Hwang, T.C., Wang, F., Yang, I.C., and Reenstra, W.W. 1997. Genistein potentiates wild-type and $\Delta$ F508-CFTR channel activity. Am. J. Physiol. 273:C988-C998.

16. Al-Nakkash, L., and Hwang, T.C. 1999. Activation of wild-type and $\Delta$ F508-CFTR by phosphodiesterase inhibitors through cAMP-dependent and -independent mechanisms. Pflügers Arch. 437:553-561.

17. Hwang, T.C., and Sheppard, D.N. 1999. Molecular pharmacology of the CFTR $\mathrm{Cl}^{-}$channel. Trends Pharmacol. Sci. 20:448-453.

18. Yang, H., et al. 2003. Nanomolar affinity small molecule correctors of defective $\triangle \mathrm{F} 508$-CFTR chloride channel gating. J. Biol. Chem. 278:35079-35085.

19. Pedemonte, N., et al. 2005. Phenylglycine and sulfonamide correctors of defective $\Delta F 508$ - and G551DCFTR chloride channel gating. Mol. Pharmacol. 67:1797-1807.

20. Ma, T., et al. 2002. Thiazolidinone CFTR inhibitor identified by high-throughput screening blocks cholera toxin-induced intestinal fluid secretion. J. Clin. Invest. 110:1651-1658. doi:10.1172/ JCI200216112.

21. Muanprasat, C., et al. 2004. Discovery of glycine hydrazide pore-occluding CFTR inhibitors: mechanism, structure-activity analysis, and in vivo efficacy. J. Gen. Physiol. 124:125-137.

22. Ma, T., et al. 2002. High-affinity activators of cystic fibrosis transmembrane conductance regulator (CFTR) chloride conductance identified by high-throughput screening. J. Biol. Chem. 277:37235-37241.

23. Kozutsumi, Y., Segal, M., Normington, K., Gething, M.J., and Sambrook, J. 1988. The presence of malfolded proteins in the endoplasmic reticulum signals the induction of glucose-regulated proteins. Nature. 332:462-464.

24. Sharma, M., et al. 2004. Misfolding diverts CFTR from recycling to degradation: quality control at early endosomes. J. Cell Biol. 164:923-933.

25. Ward, C.L., and Kopito, R.R. 1994. Intracellular turnover of cystic fibrosis transmembrane conductance regulator. Inefficient processing and rapid degradation of wild-type and mutant proteins. J. Biol. Chem. 269:25710-25718.
26. Ostedgaard, L.S., Zeiher, B., and Welsh, M.J. 1999. Processing of CFTR bearing the P574H mutation differs from wild-type and $\Delta$ F508-CFTR. J. Cell Sci. 112:2091-2098.

27. Sheppard, D.N., Ostedgaard, L.S., Winter, M.C., and Welsh, M.J. 1995. Mechanism of dysfunction of two nucleotide binding domain mutations in cystic fibrosis transmembrane conductance regulator that are associated with pancreatic sufficiency. EMBO J. 14:876-883.

28. Van Craenenbroeck, K., et al. 2005. Folding efficiency is rate-limiting in dopamine D4 receptor biogenesis. J. Biol. Chem. 280:19350-19357.

29. Gregory, R.J., et al. 1991. Maturation and function of cystic fibrosis transmembrane conductance regulator variants bearing mutations in putative nucleotide-binding domains 1 and 2. Mol. Cell. Biol. 11:3886-3893.

30. Galietta, L.J., Haggie, P.M., and Verkman, A.S. 2001. Green fluorescent protein-based halide indicators with improved chloride and iodide affinities. FEBS Lett. 499:220-224.

31. Chen, E.Y., Bartlett, M.C., Loo, T.W., and Clarke, D.M. 2004. The $\Delta$ F508 mutation disrupts packing of the transmembrane segments of the cystic fibrosis transmembrane conductance regulator. J. Biol. Chem. 279:39620-39627.

32. Johnson, L.G., et al. 1992. Efficiency of gene transfer for restoration of normal airway epithelial function in cystic fibrosis. Nat. Genet. 2:21-25.

33. Cohen, F.E., and Kelly, J.W. 2003. Therapeutic approaches to protein-misfolding diseases. Nature. 426:905-909.

34. Selkoe, D.J. 2004. Cell biology of protein misfolding: the examples of Alzheimer's and Parkinson's diseases [review]. Nat. Cell Biol. 3:1054-1056.

35. Galietta, L.J., et al. 2001. Novel CFTR chloride channel activators identified by screening of combinatorial libraries based on flavone and benzoquinolizinium lead compounds. J. Biol. Chem. 276:19723-19728.

36. Galietta, L.J., et al. 1998. An electrogenic amino acid transporter in the apical membrane of cultured human bronchial epithelial cells. Am. J. Physiol. 275:L917-L923.

37. Zhang, J.H., Chung, T.D., and Oldenburg, K.R. 1999. A simple statistical parameter for use in valuation and validation of high throughput screening assays. J. Biomol. Screen. 4:67-73. 